\title{
Use of a long-duration ns pulse for efficient emission of spectral lines from the laser ablation plume in water
}

\section{$\operatorname{AUTHOR}(S):$}

Sakka, T; Oguchi, H; Masai, S; Hirata, K; Ogata, YH; Saeki, M; Ohba, H

\section{CITATION:}

Sakka, $T$...[et al]. Use of a long-duration ns pulse for efficient emission of spectral lines from the laser ablation plume in water. APPLIED PHYSICS LETTERS 2006, 88(6): 061120.

\section{ISSUE DATE:}

2006-02-06

URL:

http://hdl.handle.net/2433/50400

\section{RIGHT:}

Copyright 2006 American Institute of Physics. This article may be downloaded for personal use only. Any other use requires prior permission of the author and the American Institute of Physics. 


\title{
Use of a long-duration ns pulse for efficient emission of spectral lines from the laser ablation plume in water
}

\author{
Tetsuo Sakka, ${ }^{a)}$ Hisayuki Oguchi, Satoru Masai, Kohichi Hirata, and Yukio H. Ogata \\ Institute of Advanced Energy, Kyoto University, Uji, Kyoto 611-0011, Japan \\ Morihisa Saeki and Hironori Ohba \\ Department of Materials Science, Japan Atomic Energy Research Institute, Tokai, Ibaraki 319-1195, Japan
}

(Received 7 October 2005; accepted 12 January 2006; published online 9 February 2006)

\begin{abstract}
The effect of pulse duration upon the line profile of $\mathrm{Cu}$ I emission observed by laser ablation of a copper metal plate immersed in water has been examined. By irradiating a pulse with the duration longer than $40 \mathrm{~ns}$ the spectral profile with clear narrow emission lines of $\mathrm{Cu}$ atoms is obtained, while the emission spectra always suffer from broadening and self-absorption by the irradiation of the 20 ns pulse for the ablation. The results show that the use of a long-duration pulse enables in situ elemental analysis of the solid surface in contact with a bulk liquid. (c) 2006 American Institute of Physics. [DOI: 10.1063/1.2172235]
\end{abstract}

Surface modifications in wet environment, such as electrochemical deposition, are important in various industrial processes. Because of the presence of water, or generally a liquid, in situ monitoring techniques of a solid surface in contact with a bulk liquid are limited to the methods which are not based on vacuum technologies. Moreover, most of them are applicable only to the surfaces with some specific conditions, e.g., surface-enhanced Raman scattering (SERS) is effective only on the SERS active surfaces, such as the surfaces coated with metallic silver. The spectrochemical analysis applicable generally to various surfaces in liquid would be useful not only for basic research but also for industry applications. We have studied laser-ablation plumes formed on a solid target immersed in liquid by emission spectroscopy. ${ }^{2-6}$ Since the ablated species are in the form of atoms and ions in the plume even in case of the ablation in liquids, ${ }^{2,3}$ an elemental analysis of the target surface should be possible, at least in principle. However, the quality of the spectra is usually too poor to enable a practical use of the emission spectra for the spectroanalytical purpose. ${ }^{3}$ The deformation of spectral line profile due to an intense emission of continuous spectrum and also the self-absorption effect caused by a high population density in the plume, ${ }^{3,5-7}$ both as a result of the strong confinement effect in water, make even qualitative analysis very difficult. For the spectrochemical analysis it is an important task to find the laser irradiation conditions which give less broadening and less selfabsorption in the emission line profile.

It has been reported that the double pulse irradiation to a solid target in water gives narrow spectral lines compared with a single pulse irradiation. ${ }^{8,}$ They used double Q-switching within a single flash of the excitation lamp for the Nd:YAG rod, obtaining double pulse with the time separation of $30-180 \mu \mathrm{s} .{ }^{8}$ The pulse energy used in their work was the order of $100 \mathrm{~mJ}$. The difference in the spectra was explained by the reheating of the plume produced by the first pulse, which forms a cavity or a bubble at the timing of the delayed second pulse; the second pulse effectively excites the gaseous state already produced in the plume by the first

\footnotetext{
a) Author to whom correspondence should be addressed; electronic mail:
} t-sakka@iae.kyoto-u.ac.jp pulse, the mechanism being similar to the gas phase laserinduced breakdown spectroscopy (LIBS) with less selfabsorbing spectra.

In the present work the effect of pulse duration upon the spectral profile was examined for a single pulse irradiation. The pulse duration was varied within the range from 20 to $150 \mathrm{~ns}$, by which we aim at the control of laser-plume interaction. It has been suggested that even in the case of the irradiation by the pulse with the duration as short as $20 \mathrm{~ns}$, the emission from the plume is already observed at the timing of the tailing edge of the ablation laser pulse. This suggests that the ablation of atoms and ions, or the plasma formation, can proceed in less than $20 \mathrm{~ns} .{ }^{4}$ Therefore, the pulse with the duration longer than $20 \mathrm{~ns}$ inevitably enhances the laser-plume interaction by the later part of a pulse. Further substantial heating of the plume is expected by using a pulse with considerably longer duration, such as several tens of ns or $100 \mathrm{~ns}$.

A home-built multimode Nd:YAG laser oscillator was operated with the Q-switch pulse mode. The fundamental wavelength of $1064 \mathrm{~nm}$ was employed. The pulse repetition rate was $\sim 0.5 \mathrm{~Hz}$. The pulse duration was controlled by adjusting the voltage of the flashlamp power source. The operation near the lasing threshold gives longer pulse duration than the operation under a higher population inversion ratio. ${ }^{10}$ The temporal pulse profiles obtained by a PIN-type silicon photodiode, of which the nominal rise time is $0.7 \mathrm{~ns}$, are given in Fig. 1 for the operation by 500, 280, and $265 \mathrm{~V}$ of the flashlamp power source. The pulse duration, which was $20 \mathrm{~ns}$ for high power operation, was considerably elongated with approaching the lasing threshold $(250 \mathrm{~V})$, and the longest pulse duration obtained with reasonable stability was $150 \mathrm{~ns}$ by our system. To obtain pulse energy adequate for the ablation the pulse from the laser oscillator was introduced into a Nd:YAG light amplifier. The final pulse energy was controlled by the pumping power of the amplifier. When the energy of the pulse before the amplifier was too high, the energy was reduced by neutral density filters.

The beam diameter of the laser immediately after the amplifier was $\sim 4 \mathrm{~mm}$. This beam was focused by a $100 \mathrm{~mm}$ focal length lens onto a copper metal plate placed horizontally in a glass cell filled with pure water. The depth from the 


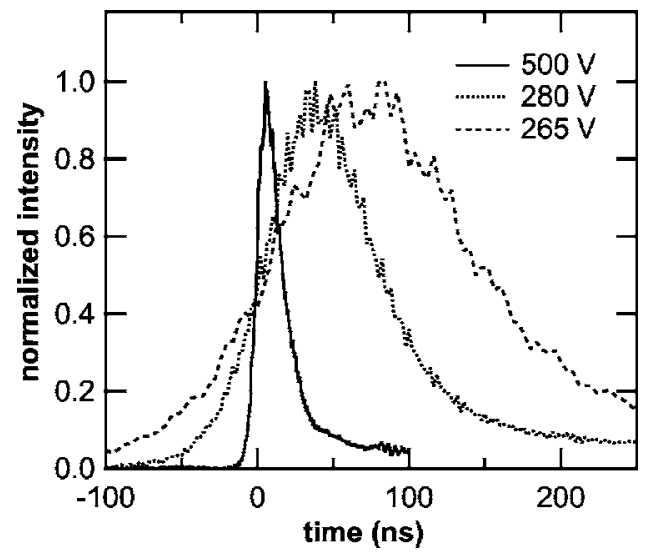

FIG. 1. Normalized temporal pulse profile for different pumping intensities. The pumping intensity was controlled by the voltage applied to the laser flashlamp.

water surface to the target surface was $\sim 5 \mathrm{~mm}$.

Lateral emission from the ablation plume was focused onto the entrance slit of a spectrograph. Since the size of the plume was so small, ${ }^{4}$ we did not perform any space resolved measurement; the focusing onto the slit was adjusted so that we obtained the maximum intensity of the emission spectra. A $27.5 \mathrm{~cm}$ focal length spectrograph (Acton, SpectraPro275) equipped with a 1200 grooves $/ \mathrm{mm}$ diffraction grating was used. The spectral range from 300 to $350 \mathrm{~nm}$ was employed for the measurement in order to cover appropriately the emission of $\mathrm{Cu} \mathrm{I}{ }^{2} S_{1 / 2}-{ }^{2} P_{3 / 2}^{o}\left(\right.$ or ${ }^{2} P_{1 / 2}^{o}$ ) fine-structure doublet at 324.75 and $327.40 \mathrm{~nm}$, respectively. ${ }^{11}$ An intensified charge coupled device (ICCD) (Princeton Instrument, ICCD1024MTDGE/1) was attached to the spectrograph, and used as a detector. The spectral resolution of this system was 0.5 $\mathrm{nm}$. The time delay from the laser pulse and the gate width to drive the ICCD detector were varied appropriately by the pulse generator (Princeton Instrument, PG-200). Each spectrum was obtained by the accumulation of the spectra measured by 5 or 10 consecutive pulse shots. The pulse repetition rate was $\sim 0.5 \mathrm{~Hz}$, and therefore, the successive pulses would never re-heat the plume produced by the former pulse.

In Fig. 2 emission spectra in the range from 318 to 333 $\mathrm{nm}$ are shown for various pulse widths. The total pulse energy of a single pulse was adjusted to $1.7 \mathrm{~mJ}$ for each measurement. This means that the peak power varies by varying the pulse duration. The gate timing of the data acquisition was set to be from $1000 \mathrm{~ns}$ after the ablation pulse to 6000 ns, i.e., the delay time of $1000 \mathrm{~ns}$ and the gate width of 5000 ns. It is clearly seen in the figure that the pulse duration longer than 40 ns gives clear emission lines with less self- absorption effects and that the lines get narrower by using a longer pulse. On the contrary, the spectrum obtained by a short pulse is deformed and broadened. The deformation is markedly seen in the spectra obtained by using a higherenergy ablation pulse in case of the pulse duration of $20 \mathrm{~ns}$. Also, the emission intensity increases with increasing pulse duration. Because the pulse energy is adjusted to the same value $(1.7 \mathrm{~mJ})$ for all the measurements in Fig. 2, the results suggest that the conversion of the input energy into the radiation of the line emission is more efficient for the laser ablation with a longer pulse. Consequently, all the results indicate that the pulse width of $150 \mathrm{~ns}$ is suitable for the elemental analysis of a solid surface in contact with a bulk liquid.

For a further examination of the pulse-duration effects upon the spectral line profile, the spectra were observed for various delay times. The pulse duration of $20 \mathrm{~ns}$ gives broadened, deformed, and also weak intensities throughout the delay time. The two emission lines are broadened and merged, and the self-reversed dips due to the self-absorption are found at the original wavelengths of the transitions. Even if we varied the pulse energy, we could never find a narrow line profile without a self-reversed structure, as long as a $20 \mathrm{~ns}$ pulse was used as an ablation laser. On the other hand, a 150 ns pulse gives a narrow emission line profile, as shown in Fig. 2, as long as very early stage of the emission, namely the delay time earlier than $400 \mathrm{~ns}$, is discarded.

The explanation of the line narrowing observed in the present work is not concrete so far. In general, a shorter pulse gives a higher ablation rate, if the pulse energy or the fluence is constant. ${ }^{12}$ This means that the higher emission intensities obtained by the elongated pulses (Fig. 2) do not indicate a higher ablation rate, but rather a higher efficiency of the emission. The efficient emission, as well as the line narrowing, seems to be attributed to a weak self-absorption, which is probably caused by the laser-plume interaction. As mentioned above, the pulse duration which gives narrow emission lines, namely the pulses with the duration longer than 40 ns, is actually long enough for the later part of the pulse to have interaction with the plume formed by the earlier part of the pulse. This may effectively heat the periphery of the plume and we can get rid of the temperature gradient causing the self-absorption. However, we are not sure up to now whether or not the further heating of the plume is actually only the cause of the line narrowing, because the heating is not expected to lower the initial high density of the plume which is the origin of the substantial line broadening. ${ }^{13}$ Despite these pending questions, we would like to point out that the interaction between the later part of the pulse and the

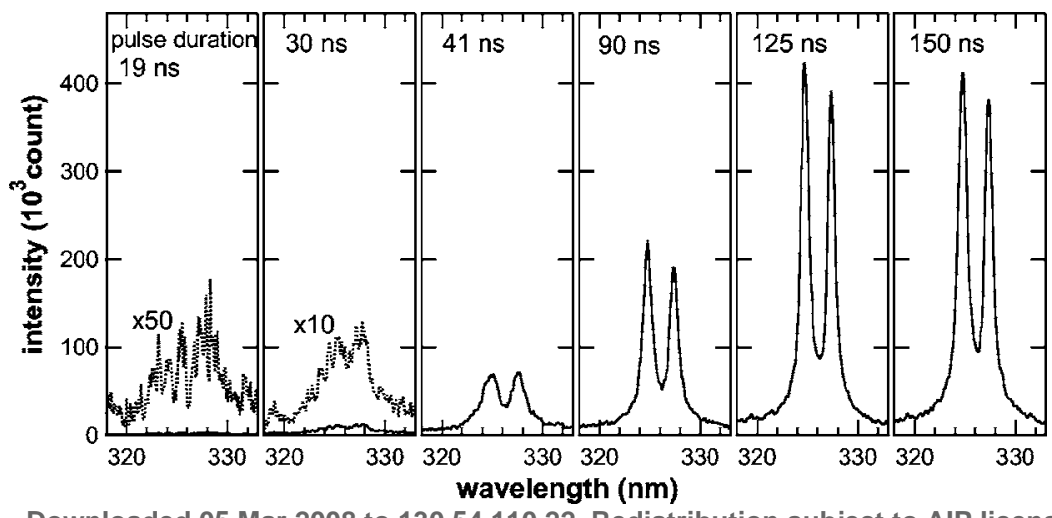

FIG. 2. Emission spectra obtained in water for the $\mathrm{Cu} \mathrm{I}$ ${ }^{2} S_{1 / 2}-{ }^{2} P_{3 / 2}^{o}\left({ }^{2} P_{1 / 2}^{o}\right)$ fine-structure doublet for various pulse durations. The energy of the ablation pulse was adjusted to $1.7 \mathrm{~mJ}$. Each spectrum is an accumulation of the spectra obtained by 10 consecutive pulse shots. 
plume is a key point to the line narrowing observed in the present work. Further work is required to explore the mechanism of this phenomenon.

The result that a low energy pulse, such as $1.7 \mathrm{~mJ}$, is enough for the observation of the clear emission lines is advantageous for the use in surface elemental analysis, since the damage on the sample surface due to the irradiation can be minimized. From this point of view the use of an elongated nanosecond pulse for in situ elemental analysis of the solid surface in liquid may preferable to the double pulse method, where the pulse energy of the order of $100 \mathrm{~mJ}$ is used.

In conclusion, we have examined the pulse-duration effects on the spectral profile of the $\mathrm{Cu}$ I emission lines at the wavelength of 324.75 and $327.40 \mathrm{~nm}$. The pulse with the duration of $20 \mathrm{~ns}$ gives broad and deformed profiles, while the narrow lines are observed with increasing the pulse duration. The results suggest that the use of an elongated pulse, such as the one with the duration of $150 \mathrm{~ns}$, is favorable for the elemental analysis of solid surface in contact with a bulk liquid.

Technical support by Kokichi Hotta is deeply acknowledged.
${ }^{1}$ J. O'M. Bockris and S. U. M. Khan, Surface Electrochemistry (Plenum, New York, 1993), p. 37.

${ }^{2}$ T. Sakka, S. Iwanaga, Y. H. Ogata, A. Matsunawa, and T. Takemoto, J. Chem. Phys. 112, 8645 (2000).

${ }^{3}$ T. Sakka, K. Takatani, Y. H. Ogata, and M. Mabuchi, J. Phys. D: Appl. Phys. 35, 65 (2002).

${ }^{4}$ K. Saito, K. Takatani, T. Sakka, and Y. H. Ogata, Appl. Surf. Sci. 197, 56 (2002).

${ }^{5}$ T. Sakka, K. Saito, and Y. H. Ogata, J. Appl. Phys. 97, 014902 (2005).

${ }^{6}$ T. Sakka, H. Oguchi, and Y. H. Ogata, Proceedings of the 8th International Conference on Laser Ablation (J. Phys.: Conference Series) (submitted).

${ }^{7}$ T. Sakka, T. Nakajima, and Y. H. Ogata, J. Appl. Phys. 92, 2296 (2002).

${ }^{8}$ A. E. Pichahchy, D. A. Cremers, and M. J. Ferris, Spectrochim. Acta B 52, 25 (1997).

${ }^{9}$ A. De Giacomo, M. Dell'Aglio, F. Colao, R. Fantoni, and V. Lazic, Appl. Surf. Sci. 247, 157 (2005).

${ }^{10}$ A. E. Siegman, Lasers (University Science Books, Mill Valley, 1986), p. 1017.

${ }^{11}$ Atomic transition data can be found in NIST atomic spectra database, http://physics.nist.gov/cgi-bin/AtData/main_asd.

${ }^{12}$ W. L. Smith, Opt. Eng. 17, 489 (1978).

${ }^{13}$ S. Masai, K. Hirata, T. Sakka, and Y. H. Ogata, Proceedings of the 8th International Conference on Laser Ablation (J. Phys.: Conference Series) (submitted). 